\title{
Construction of a high density SNP linkage map of kelp (Saccharina japonica) by sequencing Taq I site associated DNA and mapping of a sex determining locus
}

Ning Zhang ${ }^{1,2,3 \dagger}$, Linan Zhang ${ }^{4 \dagger}$, Ye Tao ${ }^{5 \dagger}$, Li Guo ${ }^{1,2,3}$, Juan Sun ${ }^{4}$, Xia Li ${ }^{4}$, Nan Zhao ${ }^{4}$, Jie Peng ${ }^{4}$, Xiaojie Li ${ }^{4^{*}}$, Liang Zeng ${ }^{5}$, Jinsa Chen ${ }^{5}$ and Guanpin Yang ${ }^{1,2,3^{*}}$

\begin{abstract}
Background: Kelp (Saccharina japonica) has been intensively cultured in China for almost a century. Its genetic improvement is comparable with that of rice. However, the development of its molecular tools is extremely limited, thus its genes, genetics and genomics. Kelp performs an alternative life cycle during which sporophyte generation alternates with gametophyte generation. The gametophytes of kelp can be cloned and crossed. Due to these characteristics, kelp may serve as a reference for the biological and genetic studies of Volvox, mosses and ferns.

Results: We constructed a high density single nucleotide polymorphism (SNP) linkage map for kelp by restriction site associated DNA (RAD) sequencing. In total, 4,994 SNP-containing physical (tag-defined) RAD loci were mapped on 31 linkage groups. The map expanded a total genetic distance of 1,782.75 cM, covering 98.66\% of the expected $(1,806.94 \mathrm{cM})$. The length of RAD tags (85 bp) was extended to 400-500 bp with Miseq method, offering us an easiness of developing SNP chips and shifting SNP genotyping to a high throughput track. The number of linkage groups was in accordance with the documented with cytological methods. In addition, we identified a set of microsatellites (99 in total) from the extended RAD tags. A gametophyte sex determining locus was mapped on linkage group 2 in a window about $9.0 \mathrm{cM}$ in width, which was 2.66 cM up to marker_40567 and 6.42 cM down to marker_23595.

Conclusions: A high density SNP linkage map was constructed for kelp, an intensively cultured brown alga in China. The RAD tags were also extended so that a SNP chip could be developed. In addition, a set of microsatellites were identified among mapped loci, and a gametophyte sex determining locus was mapped. This map will facilitate the genetic studies of kelp including for example the evaluation of germplasm and the decipherment of the genetic bases of economic traits.
\end{abstract}

Keywords: Kelp, Saccharina japonica, SNP, RAD, Linkage map, Sex determining locus

\footnotetext{
* Correspondence: yeslxj@sina.com; yguanpin@mail.ouc.edu.cn

${ }^{\dagger}$ Equal contributors

${ }^{4}$ National Engineering Science Research \& Development Center of Algae and

Sea Cucumbers of China; Provincial Key Laboratory of Genetic Improvement

\& Efficient Culture of Marine Algae of Shandong, Shandong Oriental Ocean

Sci-tech Co., Ltd, Yantai, Shandong 264003, China

'Laboratory of Marine Genetics and Breeding, Ocean University of China,

Qingdao 266003, China

Full list of author information is available at the end of the article
}

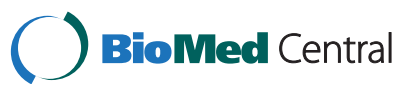

(c) 2015 Zhang et al.; licensee BioMed Central. This is an Open Access article distributed under the terms of the Creative Commons Attribution License (http://creativecommons.org/licenses/by/4.0), which permits unrestricted use, distribution, and reproduction in any medium, provided the original work is properly credited. The Creative Commons Public Domain Dedication waiver (http://creativecommons.org/publicdomain/zero/1.0/) applies to the data made available in this article, unless otherwise stated. 


\section{Background}

In Chinese aquaculture community, Saccharina japonica is referred to as kelp [1,2] although other species, e.g., giant kelp (Macrocystis pyrifera), have also been tentatively cultured in recent years. Chinese kelp breeders and farmers also call the cultured kelp either Japanese kelp or true kelp as its scientific name $S$. japonica indicated; unfortunately the cultured kelp of China may have been contaminated genetically by $S$. longissima as two interspecific hybrids $[3,4]$ and a hybrid-derived variety [5] at least have been developed and commercially cultured recently. Kelp has contributed significantly to Chinese mariculture. Its culturing area reached 40,201 hectares and its yield (weight of air dried frond) reached 979,006 tons in 2012 as was documented in Annual Report of Chinese Fisheries 2013. Kelp can be used to extracting mannitol, alginate and medicine or as human food and animal feed. Kelp promises also to be the most favorable feedstock for bioethanol fermentation as its three major components, mannitol, laminarian and alginate, can be fermented into bioethanol concertedly [6-8]. Kelp may also serve as the carbon fixers [9]. In addition, kelp has been integrated into environmental remediation and healthy animal culture systems in China.

China has cultured kelp intensively for almost a century. As were widely practiced in terrestrial crops, kelp has also been genetically improved with methods including continuous selection, hybridization and selection and development of hybrids. Since 1950s, more than 20 elite varieties and hybrids have been bred, which have contributed significantly to the culture performance of kelp, especially its yield. The representatives of these varieties included Haiqin no.1 [10], 901 [5] and Dongfang no.2 [4]. The weakness of their stress tolerance and their inapplicability for processing may not satisfy the tremendous and diverse requirements of kelp as human food, animal feed and industrial raw material; however the yield of these kelp varieties, especially that of hybrids, has been significantly elevated. Actually, the yield of hybrid kelps increases by $60-70 \%$ over normal varieties, almost a half of the theoretical, i.e. 59 metric tons/ha/year [11]. Such a margin of yield increase is more than that of hybrid rice over normal rice varieties $(\geq 20 \%)$ [12]. Kelp is the only macroalga that has received systematic genetic improvement; unfortunately the studies on its genes, genetics and genomics are far behind those of rice.

Kelp performs an alternative life cycle during which sporophyte generation alternates with gametophyte generation [13]. The sporophytes are large and multicellular while the gametophytes are microscopic, containing a single or a few cells. The asexual sporophytes (diploid) produce motile zoospores (monoploid). These zoospores develop into sexual male and female gametophytes which produce spermatozoids and eggs, respectively. Through fertilization, spermatozoids and eggs fuse to form zygotes which subsequently develop into sporophytes. Kelp gametophytes can asexually propagate [14-16], forming gametophyte clones applicable to either germplasm conservation or gametophyte clone hybridization and hybrid kelp seedling raising [3-5]. This avoids the dependence of mature sporophytes met in traditional summer seedling-raising from sporophytes [17]. Year round seedling-raising of kelp is potent as the maturing time is different among habitats [18] and physiologically modifiable [19]. The field culturing facility of kelp in China has evolved from floating raft [13] to floating rope net [3]. The seedlings were placed in between the skeins of the pendent ropes between floating head-ropes fixed at two ends to sea bed. In addition, both kelp sporophytes and gametophytes have survived the genetic transformation with diverse methods [20]. The number of chromosomes in kelp nucleus is hard to determine as they are resistant to staining with traditional chemical dyes. Accordingly, the number of chromosomes in kelp nucleus has been debated for a long time. With an improved staining method, such a number was determined as 31 by Zhou et al. [21], which was further supported by 4 , 6-diamino-2-phenyl indole (DAPI) staining [22-24]. Sex specific markers including inter-simple sequence repeat (SSR) [24] and a sequence [22] have been identified. Measurable and observable traits of kelp included at least spermatozoid life-span [25], temperature tolerance of young sporophytes [26], and those frequently evaluated during breeding, e.g. the morphological characteristics and stress tolerance [3-5]. These achievements make the intensive studies of kelp with molecular tools feasible and appreciable.

A SNP is a DNA sequence variation that occurs when a single nucleotide in the genome differs between either the members of a species or the paired chromosomes in a single individual. SNPs have gained a wide range of applications in humans and model species. They are becoming the marker of choice for additional species as well. The SNP genotyping techniques include tetra primer amplification refractory mutation system - polymerase chain reaction (T-ARMS-PCR) [27], capillary electrophoresis [28], high resolution melting [29], mass spectrometry [30,31], optical amplification of cationic conjugated polymers and the single base primer extension reaction [32], DNA microarray [33-36], pyrosequencing [37,38], multiplex PCR [39]; PCR and sequencing [40], competitive allele specific PCR (KASPar) [41], GoldenGate [42-45], resequencing [46], and among others [47]. This evolution has witnessed a dramatic increase in genotyping throughput; however the high cost, labor intensiveness and confirmation bias, alone or in combination, have slowed their application to a wide range of species and individuals within a given population. SNP genotyping by traditional DNA sequencing originally promised a high throughput, but its high cost limited its 
applicability to only model or extremely important species [48]. With the coming of the next generation (massively parallel) sequencing era, the cost of genotyping by sequencing is being progressively reduced, making the diversity determining, map constructing and trait mapping by sequencing feasible in organisms with or without reference genome sequence [49]. Implementation of methodologies such as the reduction of genome complexity [50] and restriction-site associated DNA (RAD) sequencing [36,51-53] and a modification of this method, double digest RAD (ddRAD) [54-56] has dramatically widened the application of genotyping by sequencing in linkage map construction [57,58] and association analysis [59]. In contrast, trait mapping on the SNP linkage maps constructed by sequencing has been scarce [60]. In addition to map construction and trait mapping, SNPs in chip format have also been used to genomic breeding of rice [61] and characterization of genomic diversity of wheat [62].

For kelp (S. japonica), diverse molecular markers have been developed, which included amplified fragment length polymorphism (AFLP) [63-65], SSR [66-69] and among others. These markers have been used to quantitative trait locus (QTL) mapping [70], locating gametophyte sex determining locus [64], diversity determination [71,72] and heterosis prediction [73]. However, kelp is far behind model plants and crops in the number and type of molecular markers and the applying depth and width of these markers although the breeding strategy and achievement of kelp, especially hybrid breeding, are comparable with those of rice and its potential of being a source feedstock of bioethanol fermentation. It is clear that the genetic studies of kelp should shift onto a fast molecular marker track as other plants do. In this study, a high density SNP linkage map was constructed for kelp by sequencing Taq I associated DNA, aiming to provide kelp studies an important tool.

\section{Methods}

Construction of a gametophyte clone mapping panel

The parental female gametophyte clone was isolated from Dongfang no. 3, a kelp hybrid [3], in 2004 while the parental male gametophyte clone was isolated from Benniu, a $S$. japonica variety, bred by continuous selection in 2003 . The sporophytes were raised from these parental gametophyte clones and cultured with the methods described early [4]. In Jul. 2011, three well developed mature sporophytes were selected with their heterozygosity judged by genotyping at nine microsatellites $(H 1, H 10, H 123, D 3$, D5, D9, Zspj22, Zspj28 and Zspj38) [66,68,69]. Tissue blocks with sporangia were cut from sporophytes, two each, which were scratched with cotton balls and rinsed with sterilized seawater thoroughly, submerged in $1.5 \% \mathrm{KI}$ for 5-10 min, and air-dried for 2-3 h. The blocks were submerged in sterilized seawater independently, allowing zoospores to release. The zoospores were allowed to sink and adhered onto the bottom surface of a glass dish and germinate there in a few days. The young gametophytes were picked up once their sex is distinguishable and inoculated into seawater for further growth as we did early [4]. Out of six tissue blocks, only one was selected for constructing the gametophyte clone mapping panel. The seawater used for sporophyte tissue rinsing, zoospore releasing and germinating, young gametophyte culturing and conserving and among others was filtrated through fabric ester microhole filtering films (WX-0.22 $\mu \mathrm{M}$, MOSU Instruments CO., LTD, Shanghai, China) and autoclaved at $121^{\circ} \mathrm{C}$ for $30 \mathrm{~min}$.

\section{DNA extraction}

The genomic DNA of the gametophyte clones of mapping panel and 2 parental gametophyte clones was extracted primarily with a modified cetyltriethylammnonium bromide (CTAB) method [74]. About $1.0 \mathrm{~g}$ of gametophytes was ground into powder in liquid nitrogen. To the powder,

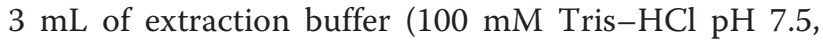
$50 \mathrm{mM}$ ethylene diamine tetraacetic acid (EDTA) $\mathrm{pH} 4.5$, $1.5 \mathrm{M} \mathrm{NaCl}, 2 \%$ CTAB, 1\% (w/v) polyvinylpyrrolidone) was added. The mixture was shaken vigorously for $5 \mathrm{~min}$ and incubated at $55^{\circ} \mathrm{C}$ for $2 \mathrm{~h}$ in the presence of 20 units of proteinase $\mathrm{K}$, and treated with $1 / 3$ extraction buffer volume of $5 \mathrm{M} \mathrm{KAc} \mathrm{(pH} \mathrm{5.0)} \mathrm{on} \mathrm{ice} \mathrm{for} 30 \mathrm{~min}$. The cellular debris and polysaccharides were removed by centrifuging at $12,000 \mathrm{rpm}$ and $4^{\circ} \mathrm{C}$ for $15 \mathrm{~min}$. The supernatant was extracted with an equal volume of phenol: chloroform: isoamyl alcohol (25:24:1 in volume) and then with an equal volume of chloroform: isoamyl alcohol (24:1) with polysaccharides removed again by adding 0.3 volumes of absolute ethanol and an equal volume of chloroform: isoamyl alcohol (24:1). The DNA was precipitated with 0.8 volume of cold isopropanol at $-20^{\circ} \mathrm{C}$ for $1 \mathrm{~h}$, washed twice with $70 \%$ cold ethanol and dissolved in $1 \times$ TE. The DNA was further purified using Plant Genomic DNA Kit (Product No. DP305, Tiangen Biotech Co., Ltd, Beijing, China). The RNA was removed away with an appropriate amount of RNase. In case of biomass-limited, e.g. some male gametophyte clones; genomic DNA was extracted directly with Plant Genomic DNA Kit. In order to verify the segregation of the mapping panel, two parental gametophyte clones and eight randomly selected clones were subjected to microsatellite genotyping (Figure 1). The polymorphic microsatellites included D5, H45 and H123 $[68,69]$. The quality genomic DNA of the gametophyte clones of mapping panel was used for RAD sequencing.

\section{RAD sequencing and SNP linkage map construction}

RAD library constructing, indexing and pooling were done with the strategy developed for natural populations [52]. The restriction endonuclease Taq I was used to 


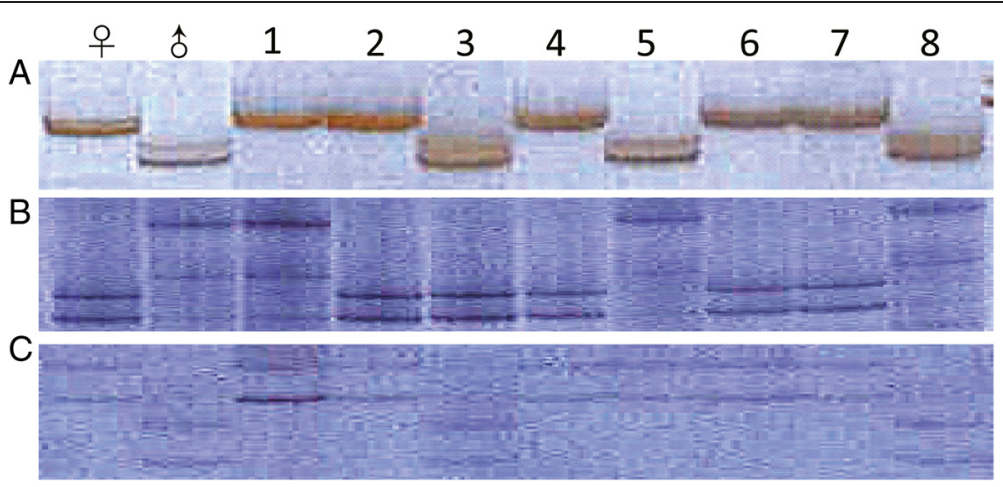

Figure 1 Microsatellite segregation pattern of mapping panel. Eight randomly selected gametophyte clones from mapping panel were genotyped with D5 (A); H45 (B) and H123 (C). The gametophyte clones of mapping panel segregate as expected.

cutting gametophyte clone DNA. A total of 24 multiplex sequencing libraries were constructed, in which each gametophyte clone DNA was prefixed with a unique nucleotide multiplex identifier as a barcode. Taq I end (101 bp in length) sequencing was performed on Illumina HiSeq2500 platform. Illumina Miseq PE250 was used to extending Taq I site associated DNA of two parental gametophyte clones. Raw RAD reads were trimmed to 85 nucleotide tags, which ensured $>97.5 \%$ of nucleotides have a quality value $>$ Q30 $(<0.1 \%$ sequencing error). These tags were aligned into $\mathrm{Taq}$ I site associated tag piles by their sequence similarity using Stacks [75]. Unique tags, i.e. the non-redundant with a maximum of one base difference from others, were screened out of a tag pile and used as the candidate alleles occupying a corresponding physical RAD locus. Physical RAD loci are sequence tagged while genetic loci are linkage determined. All candidate alleles were then collapsed into clusters using Stacks under default parameters for SNP calling [76]. Genotype calling, a process of determining the SNP genotype of physical RAD loci of each gametophyte clone after SNP calling, followed the philosophy of Hohenlohe et al. [76]. The customized perl scripts were applied then to generate a "loc" file as the input of Joinmap $3.0[77,78]$ with SNP linkage map calculated at a $\log$ of odds (LOD) value of 6.0 and a maximum recombination of 0.400 with regression algorithm. The linkage distances between loci were exported into MapChart [79] for map drawing.

The expected length of map (Ge) was the average of Ge1 and Ge2, where Ge1 was the sum of the lengths of all linkage groups, each revised by adding $2 \mathrm{~s}$ ( $\mathrm{s}$ is the average space between loci, $2 \mathrm{~s}$ accounts for the two chromosome ends) to the observed [80], and Ge2 was the sum of the lengths of linkage groups, each revised by multiplying the observed with $(m+1) /(m-1)$, where $m$ is the number of genetic loci [81]. The genome coverage was calculated by dividing the observed map length with that of the expected as we did early [82].

\section{Results}

Gametophyte clone genotyping

Trimming the raw HiSeq2500 reads yielded the quality tags for gametophyte clones each, which ranged from 341,797 to $4,028,160$ with an average of $1,905,908$. For parental female and male gametophyte clones, 5,886,795 and 5,634,477 quality tags were generated, respectively (Figure 2). The quality tags of a gametophyte clone were aligned into RAD-tag piles with those covering only one tag discarded in order to ensure sequencing reliability. The remaining RAD-tag piles were considered as physical RAD loci from which unique candidate alleles were picked up. All candidate alleles identified among gametophyte clones of mapping panel were clustered for SNP and SNP genotype calling. Of 153 gametophyte clones, 14 were deleted in linkage map calculation as they were either genotype heterozygous or genotype absent at a large portion of physical RAD loci. The remaining 139 gametophyte clones were used for map calculation.

\section{Construction and characterization of SNP linkage map}

In total, 4,994 physical RAD loci survived testing against 1:1 segregation expectation, grouping and map calculation, which were assigned onto 31 linkage groups finally

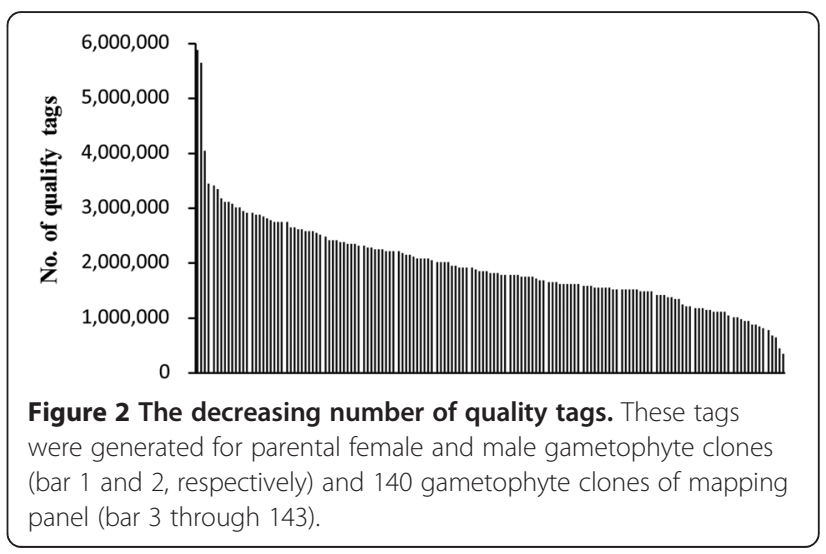


(Figure 3, Additional files 1 and 2). These physical RAD loci occupied 4,921 genetic RAD loci as some of physical RAD loci co-segregated among gametophyte clones. The number of markers was large; however their distribution was not even (Figure 3). The maximum interval between genetic RAD loci was $14.97 \mathrm{cM}$ while the minimum was $0.001 \mathrm{cM}$ with an average of $0.36 \mathrm{cM}$ (Table 1$)$. Big intervals were often found in linkage groups each, indicating that some regions of kelp genome were extremely recombination less. The length of map was 1,782.75 cM in length, which accounted for $98.66 \%$ of the expected $(1,806.94 \mathrm{cM})$.

\section{Characterization of SNP at mapped physical RAD loci}

Gametophyte clones contained different numbers of quality physical RAD loci. About $21.6 \%$ of quality physical RAD loci were absent in average among these clones. As illustrated in Figure 3, the number of mapped physical RAD loci varied among clones. As showed in Figure 4, only seven clones were genotyped at $<3,000$ physical RAD loci, and 63 clones were genotyped at >5,000 physical RAD loci. The remaining clones were genotyped at physical RAD loci varying between 3,000 and 5,000. All types of SNP were identified at mapped physical loci. Along 85 nucleotide tags, SNP distributed almost evenly (Figure 5).

\section{Extension of Taq I associated DNA and microsatellites development}

At 4,992 of 4,994 physical RAD loci, Taq I associated DNA was extended with Illumina Miseq PE250. The number of trimmed extending reads at these loci ranged from 552 to 4,677 with an average of 897 (Additional file 3). These extended DNA will expand the application of these SNPs to e.g. the development of oligonucleotide chips.

The extended tags have allowed us to develop a set of microsatellites for determining genetic diversity, evaluating genetic resources and among others. Microsatellites searching against extended Taq I associated DNA at 4,992 physical RAD loci yielded 99 microsatellites (Additional file 4). The simple sequence of these microsatellites was 2-6 bases in length and repeated $\geq 10(2 n), \geq 7(3 n), \geq 5$ (4n), $\geq 4$ (5n) and $\geq 3$ (6n) times, respectively. These microsatellites were bounded at least 50 nucleotides upstream and downstream, respectively, so that primers can be designed conveniently. Unfortunately, the amplification and polymorphism between two parental gametophyte clones and among conserved kelp gametophyte clones have not been tested. The positions of the Taq I associated DNA from them the microsatellites were identified have added locating the microsatellites at the SNP linkage map constructed in this study (Additional files 2 and 4 in red color).

\section{Mapping of a gametophyte sex determining locus}

After calculating SNP linkage map, we mixed the sex trait of kelp gametophyte clones with SNP genotypes of physical RAD loci, grouped these loci and sex trait again and calculated the linkage of gametophyte sex trait and physical RAD loci. It was found that a sex determining locus was mapped on linkage group 2, which was $2.66 \mathrm{cM}$ up to marker_40567 and $6.42 \mathrm{cM}$ down to marker_23595 (Additional files 5 and 6 in red color). Linkage group 2 was the longest, expanding a genetic distance of $92.6 \mathrm{cM}$. Recalculated linkage group containing sex determining locus was $93.0 \mathrm{cM}$ in length. The sex determining locus located within a window of about $9.0 \mathrm{cM}$ in length.

\section{Discussion}

\section{SNP linkage map and its application}

Kelp has been intensively cultured in China for almost a century. Its genetic improvement is comparable with that of rice in terms of breeding strategy. For example, utilizing heterosis by developing kelp hybrids has increased the unit area yield by $60-70 \%$ on the basis of elite varieties [3-5]. In addition, a set of traits, both the biological (e.g. gametophyte gender) and the economic (e.g. yield and stress tolerance) have been measured frequently during breeding. It is clear that the genetic bases of these traits should be deciphered with molecular tools so that molecular marker assisted breeding could be implemented. Unfortunately, the development of molecular tools for kelp is extremely limited. A few marker systems have been used in kelp [63-69]; however they were either low throughput or not transferable among populations or number limited. Linkage maps of kelp have been tentatively constructed (e.g., [64]); unfortunately the markers on these maps are mainly AFLPs which are not transferable among populations.

In this study, we constructed a high density SNP linkage map by sequencing Taq I site associated DNA. In total, 4,994 SNPs were assigned to 31 linkage groups. The map expanded a total genetic distance of $1782.75 \mathrm{cM}$, covering $98.66 \%$ of the expected (1806.94 cM). To our knowledge, this is the highest density molecular marker linkage map of kelp constructed to date. The length of RAD tags were 85 bp in length, These tags themselves may serve as interrogators of their homologs; however they cannot serve as the templates of SNP detecting tools, for example, PCR based methods. In order to overcome this shortage, we extended the tag to $400-500 \mathrm{bp}$ with Miseq method. The extended will retain the advantage of tags in homologs searching and offer us easiness in developing SNP detecting tools, and further deciphering the genetic bases of economic traits and cloning their controlling genes. The number of linkage groups we obtained was in accordance with that determined with an improved staining method [21] and DAPI staining [22-24]. Sex specific markers including inter-SSRs [24] and a sequence [22] have been identified. The mapping of kelp gametophyte sex locus and these early findings in combination may 


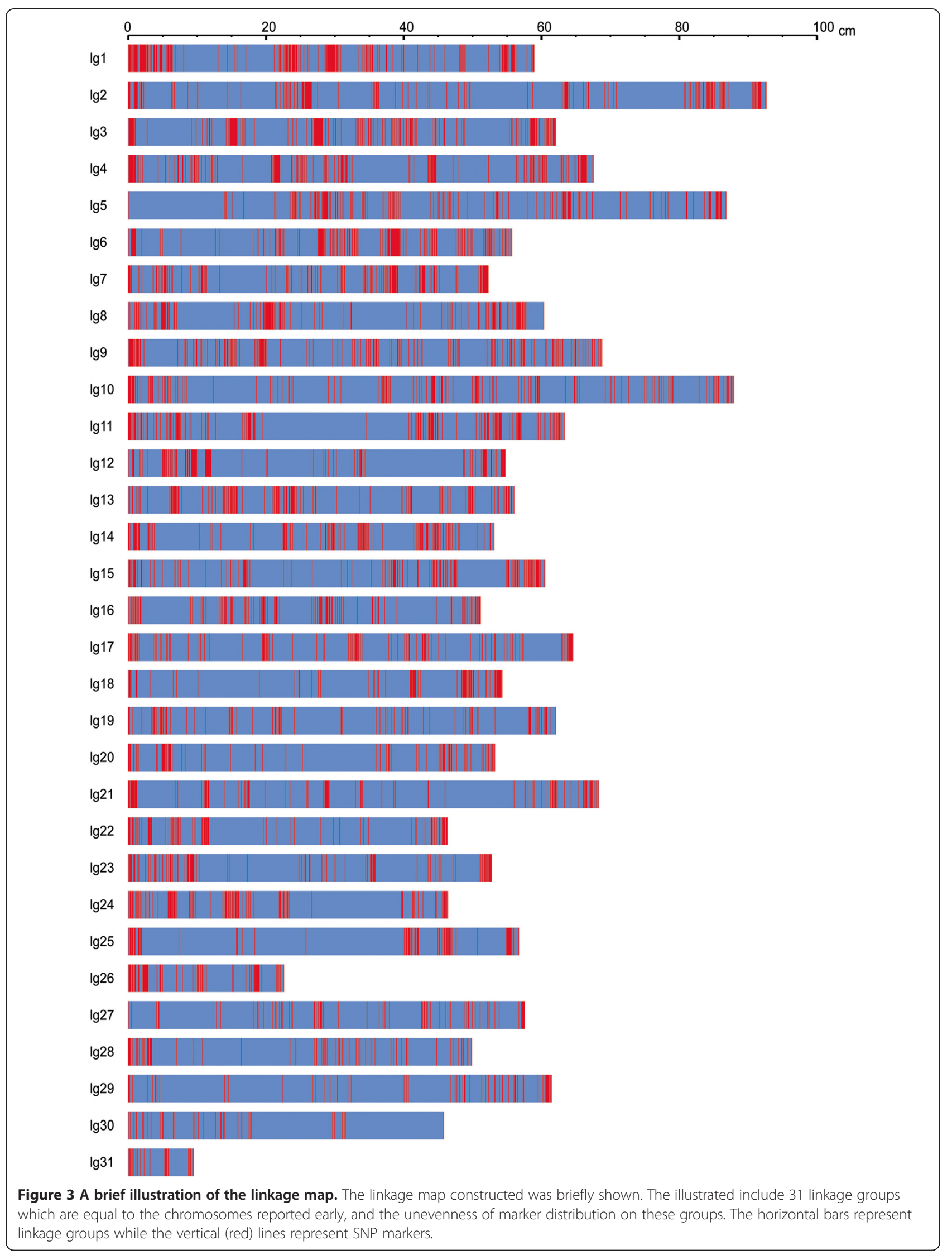


Table 1 The characteristics of the linkage map constructed in this study

\begin{tabular}{|c|c|c|c|c|c|c|c|c|}
\hline \multirow[t]{2}{*}{ LG } & \multicolumn{2}{|l|}{ No. of } & \multicolumn{3}{|c|}{ Space (cM) } & \multicolumn{3}{|c|}{ Length (cM) } \\
\hline & Markers & Loci & Max. & Min. & Avr. & Cal. & Rev.1 & Rev.2 \\
\hline 1 & 308 & 304 & 5.123 & 0.001 & 0.195 & 58.913 & 59.638 & 59.303 \\
\hline 2 & 279 & 262 & 9.846 & 0.001 & 0.356 & 92.619 & 93.344 & 93.331 \\
\hline 3 & 278 & 276 & 6.530 & 0.001 & 0.226 & 62.056 & 62.781 & 62.509 \\
\hline 4 & 268 & 268 & 8.258 & 0.001 & 0.254 & 67.532 & 68.257 & 68.040 \\
\hline 5 & 252 & 249 & 13.957 & 0.001 & 0.351 & 86.804 & 87.529 & 87.507 \\
\hline 6 & 227 & 225 & 5.008 & 0.001 & 0.250 & 55.674 & 56.399 & 56.173 \\
\hline 7 & 220 & 216 & 6.874 & 0.001 & 0.244 & 52.262 & 52.987 & 52.750 \\
\hline 8 & 217 & 211 & 8.349 & 0.001 & 0.289 & 60.329 & 61.054 & 60.906 \\
\hline 9 & 197 & 197 & 4.893 & 0.001 & 0.353 & 68.765 & 69.490 & 69.470 \\
\hline 10 & 192 & 192 & 6.211 & 0.001 & 0.463 & 87.906 & 88.631 & 88.831 \\
\hline 11 & 190 & 188 & 14.969 & 0.001 & 0.340 & 63.319 & 64.044 & 64.000 \\
\hline 12 & 183 & 178 & 14.209 & 0.001 & 0.311 & 54.736 & 55.461 & 55.358 \\
\hline 13 & 168 & 169 & 4.506 & 0.001 & 0.335 & 56.004 & 56.729 & 56.675 \\
\hline 14 & 167 & 165 & 6.576 & 0.001 & 0.326 & 53.105 & 53.830 & 53.757 \\
\hline 15 & 166 & 166 & 7.178 & 0.003 & 0.369 & 60.478 & 61.203 & 61.216 \\
\hline 16 & 137 & 138 & 7.021 & 0.001 & 0.376 & 51.134 & 51.859 & 51.886 \\
\hline 17 & 136 & 137 & 5.583 & 0.002 & 0.478 & 64.559 & 65.284 & 65.515 \\
\hline 18 & 137 & 134 & 8.914 & 0.001 & 0.411 & 54.272 & 54.997 & 55.094 \\
\hline 19 & 130 & 130 & 6.799 & 0.001 & 0.485 & 62.051 & 62.776 & 63.021 \\
\hline 20 & 128 & 128 & 10.776 & 0.001 & 0.422 & 53.204 & 53.929 & 54.049 \\
\hline 21 & 125 & 126 & 10.013 & 0.001 & 0.551 & 68.287 & 69.012 & 69.388 \\
\hline 22 & 124 & 124 & 7.900 & 0.001 & 0.380 & 46.314 & 47.039 & 47.073 \\
\hline 23 & 124 & 123 & 7.471 & 0.001 & 0.436 & 52.733 & 53.458 & 53.605 \\
\hline 24 & 121 & 122 & 13.093 & 0.001 & 0.387 & 46.385 & 47.110 & 47.158 \\
\hline 25 & 104 & 104 & 14.271 & 0.001 & 0.556 & 56.689 & 57.414 & 57.801 \\
\hline 26 & 96 & 96 & 3.828 & 0.004 & 0.240 & 22.565 & 23.290 & 23.045 \\
\hline 27 & 85 & 86 & 8.327 & 0.002 & 0.685 & 57.517 & 58.242 & 58.886 \\
\hline 28 & 84 & 85 & 7.183 & 0.002 & 0.601 & 49.865 & 50.590 & 51.067 \\
\hline 29 & 82 & 83 & 9.39 & 0.002 & 0.759 & 61.439 & 62.164 & 62.956 \\
\hline 30 & 40 & 41 & 14.250 & 0.025 & 1.174 & 45.786 & 46.511 & 48.134 \\
\hline 31 & 29 & 29 & 2.886 & 0.003 & 0.350 & 9.445 & 10.170 & 10.145 \\
\hline Total & 4994 & 4922 & 14.969 & 0.001 & 0.362 & 1782.747 & 1805.222 & 1808.649 \\
\hline
\end{tabular}

LG, linkage group; Loci refer to genetic ones, not sequence tagged physical RAD loci; Max, maximum; Min, minimum; Avr., Average; Cal., calculated length of a linkage group; Rev. 1, the length of a linkage group revised with the method of Fishman et al. [80]; Rev. 2, the length of a linkage group revised with the method of Chakravarti et al. [81].

aid to approaching the mechanism underlining the sex determination of kelp gametophytes.

SNP genotyping is less efficient with traditional methods, e.g. tetra-primer ARMS PCR [27], capillary electrophoresis [28], high resolution melting [29], mass spectrometry [30,31], optical amplification of cationic conjugated polymers and the single base primer extension reaction [32], and PCR and sequencing [40]. Such efficiency may be improved with relatively high throughput methods, e.g. multiplex PCR [39], KASPar [41] and GoldenGate [42-45]; however these methods need specifically developed facilities and detergents. Pyrosequencing [37,38], resequencing [46] and even the reduction of genome complexity [50] and RAD sequencing [36,51-53] and ddRAD [54-56,83] are still expensive at this moment. To our knowledge, DNA microarray [33-36] is the most appropriate tool for SNP genotyping as was tried in genomic breeding of rice [61] and characterization of genomic 


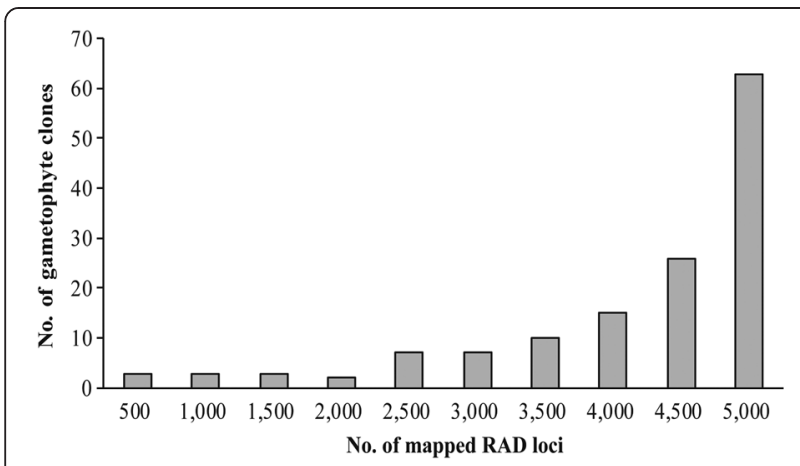

Figure 4 The number of gametophyte clones with different numbers of mapped RAD loci.

diversity of wheat [62]. We mapped 4,994 RAD physical loci and extended these tags to 400-500 bp in length. This will allow us easiness of developing SNP chips, thus shifting SNP genotyping to a fast, cheap and high throughput track.

Although a long time effort has been made, the kelp microsatellites isolated and mapped were extremely scarce $[23,66,67,69]$. In this study, we identified a set of microsatellites, 99 in total, from the mapped and extended RAD tags following relatively strict standards, which distributed on all SNP linkage groups (Additional files 2 and 4 in red color). These microsatellites will certainly fill in the gap between traditional molecular markers and high throughput SNP array or SNP chip, thus facilitating a set of works, for example, the evaluation of genetic resources and decipherment of the genetic bases of important traits. The most prominent advantage of this set of microsatellites was the certainty of their position on the linkage map.

\section{Sex determination of kelp gametophyte}

Different sets of genes governing the biosyntheses of ethylene, jasmonic acid, brassinosteroids or gibberellins or occasionally proteins have been identified as the controller of the gender of monoecious plant flowers while a sex determining region (locus) usually evolves to control the gender of dioecious plants [84]. The complexity of sex determining regions varied among a wide range of species between the simplest in yeast where only a gene and its expression regulation elements exist [85] and the most complex, the sex chromosomes in papaya [86-88]. Between the extremes of complexities are sex-determining loci governing the gender of Chlamydomonas [89,90], Volvox [91,92] and asparagus [93]. The characteristics shared by these loci include the chromosomal rearrangement and avoidance of genetic recombination and the difference in gene content and expression between genders [92]. The life cycles of liverwort and moss are monoploidy gametophytes dominant. In these haploidy systems, sex chromosomes have evolved $[94,95]$. Pheromone involve in the recognition and fusion of algal gametes, but not the gamete development and sex determination [96]. Fungi belonged to the unikonts, a supergroup of eukaryotes including animals and humans ourselves while green algae belonged to the plantae; and brown algae belonged to chromalveolates [97,98]. The complexity of sex determining loci varies among and within these eukaryotic supergroups.

Eukaryotic analyses have showed that the sex determining locus containing chromosomes (sex chromosomes) of kelp are morphologically identical [21-24]. In this study, a sex determining locus was mapped on linkage group 2 in a window about $9.0 \mathrm{cM}$ in width $(2.66 \mathrm{cM}$ up to marker_40567 and $6.42 \mathrm{cM}$ down to marker_23595). Mapping of the sex determining locus of kelp gametophytes stepped one pace at least toward the structure of this locus and its comparison with those of other species. Recently, the structure of the sex determination locus of model brown alga Ectocarpus has been described [99]. Fine mapping this locus and describing its structure thus understanding the sex determination of kelp gametophytes will be the focus of our future studies. Although a long way ahead, we will model our future works on what have achieved in Ectocarpus.

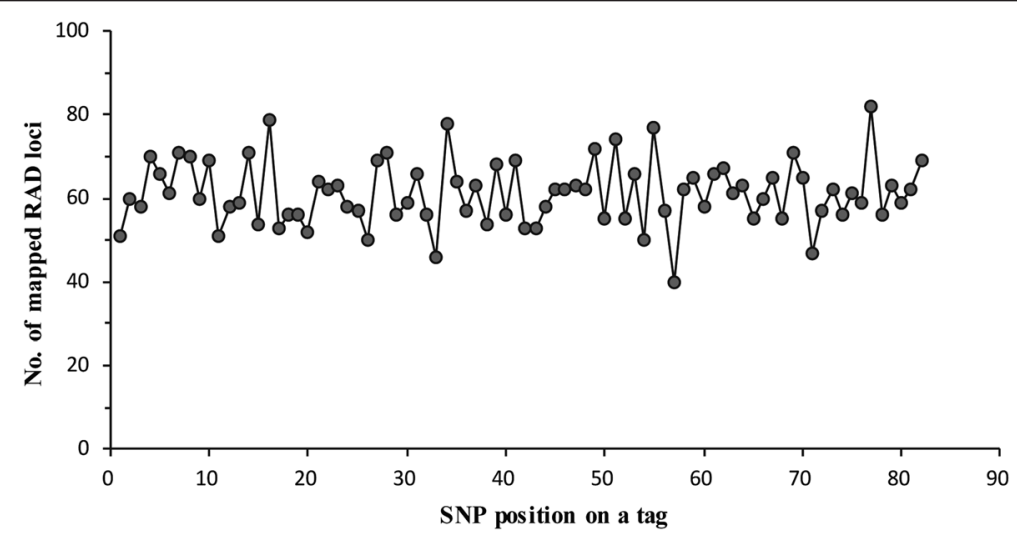

Figure $\mathbf{5}$ The number of SNPs found at different nucleotide positions of RAD tags. 


\section{Conclusions}

A high density SNP linkage map was constructed for kelp, an intensively cultured brown alga in China. On 31 linkage groups, 4,994 SNP-containing tag defined RAD loci were mapped. The map expanded a total distance of $1,782.75 \mathrm{cM}$, covering $98.66 \%$ of the expected. The number of linkage groups was in accordance with that of real chromosomes. The length of RAD tags was extended to about 400-500 bp so that SNP based tools, e.g. SNP chips, can be developed. In addition, 99 microsatellites were identified among extended RAD tags. A sex determining locus was mapped on linkage group 2. This map will facilitate the studies on kelp genes, genetics and genomics, and may provide a reference for those studies in Volvox, mosses and ferns.

\section{Availability of supporting data}

The RAD reads of 139 gametophytes as a mapping panel and 2 parental gametophytes of kelp (Saccharina japonica) have been submitted to NCBI under a bioproject accession number PRJNA274218 (http://www.ncbi.nlm.nih.gov/bioproject/274218) which links to 141 Sequence Read Archive (SRA) accessions corresponding to 141 gametophytes, respectively. The mapped RAD tags with SNP marked have also been listed in Additional file 1. The extensions of mapped RAD tags have been listed in Additional file 3.

\section{Additional files}

Additional file 1: Tabulated SNP linkage map constructed in this study. The name of markers, the sequence of RAD tags, and the recombination value between markers are listed.

Additional file 2: Graphical SNP linkage map constructed in this study with the loci containing microsatellites marked red. The mapped SNP markers, their position on linkage groups and genetic distance in $\mathrm{CM}$ are illustrated.

Additional file 3: Tag sequences extended with Miseq. In order to amplify the applicability of the map constructed to, for example, the development of SNP genotyping tools other than sequencing, the mapped RAD tags are extended. The listed include the extended tag sequences and their corresponding marker names.

Additional file 4: Microsatellites identified in extended tag sequences and their characteristics. In total, 99 microsatellites were identified among the extended tag sequences. These microsatellites are applicable to diverse studies as their location on the map is known.

Additional file 5: Tabulated position of the sex determining locus of kelp gametophytes. In this table, the sex determining locus and its position on linkage group 2 are listed.

Additional file 6: Graphical position of the sex determining locus of kelp gametophytes. The illustrated include the sex determining locus (in red color) and its relationship to the adjacent markers on linkage group 2.

\section{Abbreviations}

SNP: Single nucleotide polymorphism; RAD: Restriction site associated DNA; DAPI: 4,6-diamino-2-phenyl indole; SSR: Simple sequence repeat; T-ARMSPCR: Tetra primer amplification refractory mutation system - polymerase chain reaction; KASPar: Competitive allele specific PCR; ddRAD: Double digest RAD; AFLP: Amplified fragment length polymorphism; QTL: Quantitative trait locus; CTAB: Cetyltriethylammnonium bromide; EDTA: Ethylene diamine tetraacetic acid; LOD: Log of odds; BAC: Bacterial artificial chromosome.

\section{Competing interests}

The authors declare that they have no competing interests.

\section{Authors' contributions}

NiZ, LNZ and YT prepared DNA and constructed linkage map; LG made statistics of the map constructed and edited figures and tables; JS, XL, NaZ, JP and XJL created and maintained the mapping panel; LZ and JSC constructed libraries and obtained tag sequences; and GPY applied found, organized the study and wrote manuscript. All authors read and approved the final manuscript.

\section{Acknowledgements}

This work was financially supported by National High Technology Research and Development Program (863 Program) of China (2012AA10A406) and National Science and Technology Supporting Program of China (2012BAD55G01). The authors greatly appreciate the assistances of Zhuangzhi Zhang, Guangjing Liang and Shaofeng Song, Shandong Oriental Ocean Sci-tech Co., Ltd., Yantai, China, in seedling raising and sporophyte field culturing.

\section{Author details}

'Laboratory of Marine Genetics and Breeding, Ocean University of China, Qingdao 266003, China. ${ }^{2}$ Institute of Evolution and Marine Biodiversity, Ocean University of China, Qingdao 266003, China. ${ }^{3}$ College of Marine Life Sciences, Ocean University of China, Qingdao 266003, China. ${ }^{4}$ National Engineering Science Research \& Development Center of Algae and Sea Cucumbers of China; Provincial Key Laboratory of Genetic Improvement \& Efficient Culture of Marine Algae of Shandong, Shandong Oriental Ocean Sci-tech Co., Ltd, Yantai, Shandong 264003, China. ${ }^{5}$ Majorbio Pharm Technology Co., Ltd, Shanghai 201203, China.

Received: 16 October 2014 Accepted: 20 February 2015

Published online: 15 March 2015

\section{References}

1. Bartsch I, Wiencke C, Bischof K, Buchholz CM, Buck BH, Eggert A, et al. The genus Laminaria sensu lato: recent insights and developments. Eur J Phycol. 2008:43:1-86.

2. Lane CE, Mayes C, Druehl LD, Saunders GW. A multi-gene molecular investigation of the kelp (Laminariales, Phaeophyceae) supports substantial taxonomic re-organization. J Phycol. 2006:42:493-512.

3. Li XJ, Liu JL, Cong YZ, Qu SC, Zhang ZZ, Dai HL, et al. Breeding and trial cultivation of Dongfang no.3, a hybrid of Laminaria gametophyte clones with a more than intraspecific less than interspecific relationship. Aquaculture. 2008;280:76-80.

4. Li XJ, Cong YZ, Yang GP, Shi YY, Qu SC, Li ZL, et al. Trait evaluation and trial cultivation of Dongfang no. 2, the hybrid of a male gametophyte clone of Laminaria longissima (Laminariales, Phaeophyta) and a female one of $L$. japonica. J Appl Phycol. 2007;19:139-51.

5. Zhang QS, Tang XX, Cong YZ, Qu SC, Luo SJ, Yang GP. Breeding of an elite Laminaria variety $90-1$ through inter-specific gametophyte crossing. J Appl Phycol. 2007;19:303-11.

6. Kraan S. Mass-cultivation of carbohydrate rich macroalgae, a possible solution for sustainable biofuel production. Mitig Adapt Strat Glob Chang. 2013;18:27-46

7. Wargacki AJ, Leonard E, Win MN, Regitsky DD, Santos CNS, Kim PB, et al. An engineered microbial platform for direct biofuel production from brown macroalgae. Science. 2012;335:308-13.

8. Takeda H, Yoneyama F, Kawai S, Hashimoto W, Murata K. Bioethanol production from marine biomass alginate by metabolically engineered bacteria. Energy Environ Sci. 2011;4:2575-81.

9. Gao K, McKinley KR. Use of macroalgae for marine biomass production and CO2 remediation: a review. J Appl Phycol. 1994;6:45-60.

10. Fang TC, Wu CY, Jiang BY, Li JJ, Ren GZ. The breeding of a new breed of Haidai (Laminaria japonica Aresch.) and its preliminary genetic analysis. Acta Botanica Sinica. 1962;10:197-209 (in Chinese with English abstract).

11. Roesijadi G, Jones SB, Snowden-Swan LJ, Zhu Y. Macroalgae as a biomass feedstock: a preliminary analysis, PNNL-19944. Richland, WA: Pacific Northwest National Laboratory; 2010.

12. Li J, Yuan L. Hybrid rice: genetics, breeding, and seed production. In: Janick J, editor. Plant breeding reviews. Volume 17. Oxford: John Wiley \& Sons, Inc; 2010. p. 15-158. 
13. Scoggan J, Zhuang Z, Wang F. Culture of Kelp (Laminaria japonica) in China, RAS/86/024. Qingdao, China: Yellow Sea Fisheries Research Institute; 1989. p. 1989.

14. Zhou Z, Li D, Wu C, Liu H. Laminaria gametophyte clone culture and its application in sporeling cultivation. Acta Oceanologica Sinica. 2000;19:89-95.

15. Zhang QS, Qu SC, Cong YZ, Luo SJ, Tang XX. High throughput culture and gametogenesis induction of Laminaria japonica gametophyte clones. J Appl Phycol. 2008;20:205-11.

16. Xu B, Zhang QS, Qu SC, Cong YZ, Tang XX. Introduction of a seedling production method using vegetative gametophytes to the commercial farming of Laminaria in China. J Appl Phycol. 2009;21:171-8.

17. Tseng CK, Sun KY, Wu CY. On the cultivation of Haitai (Laminaria japonica Aresch.) by summering young sporophytes at low temperature. Acta Botanica Sinica. 1955:4:255-64 (in Chinese with English abstract)

18. Forbord S, Skjermo J, Arff J, der Handå A, Reitan Kl, Bjerregaard R, et al. Development of Saccharina latissima (Phaeophyceae) kelp hatcheries with year-round production of zoospores and juvenile sporophytes on culture ropes for kelp aquaculture. J Appl Phycol. 2012;24:393-9.

19. Pang S, Lüning K. Breaking seasonal limitation: year-round sporogenesis in the brown alga Laminaria saccharina by blocking the transport of putative sporulation inhibitors. Aquaculture. 2004;240:531-41.

20. Qin S, Jiang P, Tseng CK. Molecular biotechnology of marine algae in China. Hydrobiologia. 2004;512:21-6.

21. Zhou LR, Dai JX, Shen SD. An improved chromosome preparation from male gametophyte of Laminaria japonica (Heterokontophyta). Hydrobiologia. 2004;512:141-4.

22. Liu Y, Bi YH, Gu JG, Li LH, Zhou ZG. Localization of a female-specific marker on the chromosomes of the brown seaweed Saccharina japonica using fluorescence in situ hybridization. PLoS One. 2012;7:e48784.

23. Liu Y, Bi YH, Zhou ZG. Karyological observation on Saccharina japonica chromosomes stained with DAPI. J Fisheries China. 2012;36:50-4 (in Chinese with English abstracy).

24. Gu JG, Sun YP, Liu Y, Bi YH, Zhou ZG. Sex identification and genetic variation of Saccharina (Phaeophyta) gametophytes as revealed by inter-simple sequence repeat (ISSR) markers. J Appl Phycol. 2014;26:635-46.

25. Li J, Pang SJ, Liu F, Shan TF, Gao SQ. Spermatozoid life-span of two brown seaweeds, Saccharina japonica and Undaria pinnatifida, as measured by fertilization efficiency. Chinese J Oceanol Limnol. 2013;31:774-81.

26. Pang SJ, Jin ZH, Sun JZ, Gao SQ. Temperature tolerance of young sporophytes from two populations of Laminaria japonica revealed by chlorophyll fluorescence measurements and short-term growth and survival performances in tank culture. Aquaculture. 2007;262:493-503.

27. Ye S, Dhillon S, Ke X, Collins AR, Day IN. An efficient procedure for genotyping single nucleotide polymorphisms. Nucleic Acids Res. 2001;29:E88-8.

28. Drabovich AP, Krylov SN. Identification of base pairs in single-nucleotide polymorphisms by MutS protein-mediated capillary electrophoresis. Anal Chem. 2006;78:2035-8.

29. Li F, Niu B, Huang Y, Meng Z. Application of high-resolution DNA melting for genotyping in lepidopteran non-model species: Ostrinia furnacalis (Crambidae). PLoS One. 2012;7:e29664.

30. Tripathi N, Hoffmann M, Willing EM, Lanz C, Weigel D, Dreyere C. Genetic linkage map of the guppy, Poecilia reticulata, and quantitative trait loci analysis of male size and color variation. Proc R Soc B Biol Sci. 2009:276:2195-208.

31. Griffin TJ, Smith LM. Genetic identification by mass spectrometric analysis of single nucleotide polymorphisms: Ternary encoding of genotypes. Analytical Chemistr. 2000;72:3298-302.

32. Duan XR, Yue W, Liu LB, Li ZP, Li YL, He FC, et al. Single-nucleotide polymorphism (SNP) genotyping using cationic conjugated polymers in homogeneous solution. Nat Protoc. 2009;4:984-91.

33. Akond M, Liu S, Schoener L, Anderson JA, Kantartzi SK, Meksem K, et al. A SNP-based genetic linkage map of soybean using the SoyS-NP6K Illumina Infinium BeadChip Genotyping Array. J Plant Genome Sci. 2013;2013(1):80-9.

34. Raman H, Dalton-Morgan J, Diffey S, Raman R, Alamery S, Edwards D, et al. SNP markers-based map construction and genome-wides linkage analysis in Brassica napus. Plant Biotechnol J. 2014; doi: 10.1111/ pbi.12186.

35. Fu $Y$, Springer NM, Ying $K$, Yeh $\subset$, Iniguez AL, Richmond $T$, et al. High-resolution genotyping via whole genome hybridizations to microarrays containing long oligonucleotide probes. PLoS One. 2010;5:e14178.

36. Miller MR, Atwood TS, Eames BF, Eberhart JK, Yan YL, Postlethwait JH, et al. RAD marker microarrays enable rapid mapping of zebrafish mutations. Genome Biol. 2007;8:R105.
37. Royo JL, Hidalgo M, Ruiz A. Pyrosequencing protocol using a universal biotinylated primer for mutation detection and SNP genotyping. Nat Protoc. 2007;2:1734-9.

38. Wang $\mathrm{H}$, Elbein SC. Detection of allelic imbalance in gene expression using pyrosequencing. Methods Mol Biol. 2007;373:157-75

39. Foley BR, Rose CG, Rundle DE, Leong W, Moy GW, Burton RS, et al. A gene-based SNP resource and linkage map for the copepod Tigriopus californicus. BMC Genomics. 2011;12:568.

40. Yamamoto K, Narukawa J, Kadono-Okuda K, Nohata J, Sasanuma M, Suetsugu $Y$, et al. Construction of a single nucleotide polymorphism linkage map for the silkworm, Bombyx mori, based on bacterial artificial chromosome end sequences. Genetics. 2006;173:151-61.

41. Hiremath PJ, Kumar A, Penmetsa RV, Farmer A, Schlueter JA, Chamarthi SK, et al. Large-scale development of cost-effective SNP marker assays for diversity assessment and genetic mapping in chickpea and comparative mapping in legumes. Plant Biotechnol J. 2012;10:716-32.

42. Bradley KM, Breyer JP, Melville DB, Broman KW, Knapik EW, Smith JR. An SNP-based linkage map for zebrafish reveals sex determination loci. Genes Genomes Genetics. 2011;1:3-9.

43. Hubert S, Higgins B, Borza T, Bowman S. Development of a SNP resource and a genetic linkage map for Atlantic cod (Gadus morhua). BMC Genomics. 2010;11:191.

44. Kenta S, Sachiko I, Hideki H, Erika A, Hiroyuki F, Daniel J, et al. SNP Discovery and linkage map construction in cultivated tomato. DNA Res. 2010;17:381-91.

45. Rashmi G, Sarwar A, Ganga J, Aamiir Waseem K, Shalu C, Mukesh J, et al. High-throughput SNP discovery and genotyping for constructing a saturated linkage map of chickpea (Cicer arietinum L.). DNA Res. 2012;19:357-73.

46. Shi YY, Sun LX, Huang ZY, Wu XB, Zhu YQ, Zheng HJ, et al. A SNP based high-density linkage map of Apis cerana reveals a high recombination rate similar to Apis mellifera. PLoS One. 2013;8:e76459.

47. Garvin MR, Saitoh K, Gharrett AJ. Application of single nucleotide polymorphisms to non-model species: A technical review. Mol Ecol Resour. 2010;10:915-34

48. Altshuler D, Pollara VJ, Cowles CR, van Etten WJ, Baldwin J, Linton L, et al. An SNP map of the human genome generated by reduced representation shotgun sequencing. Nature. 2000;407:513-6.

49. Willing EM, Hoffmann M, Klein JD, Weigel D, Dreyer C. Paired-end RAD-seq for de-novo assembly and marker design without available reference. Bioinformatics. 2011:27:2187-93.

50. Sánchez CC, Smith TPL, Wiedmann RT, Vallejo RL, Salem M, Yao JB, et al. Single nucleotide polymorphism discovery in rainbow trout by deep sequencing of a reduced representation library. BMC Genomics. 2009;10:559.

51. Miller MR, Dunham JP, Amores A, Cresko WA, Johnson EA. Rapid and cost-effective polymorphism identification and genotyping using restriction site associated DNA (RAD) markers. Genome Res. 2007;17:240-8.

52. Baird NA, Etter PD, Atwood TS, Currey MC, Shiver AL, Lewis ZA, et al. Rapid SNP discovery and genetic mapping using sequenced RAD markers. PLoS One. 2008;3:e3376,

53. Baxter SW, Davey JW, Johnston JS, Shelton AM, Heckel DG, Jiggins CD, et al. Linkage mapping and comparative genomics using next-generation RAD sequencing of a non-model organism. PLoS One. 2011;6:e19315.

54. Peterson BK, Weber JN, Kay EH, Fisher HS, Hoekstra HE. Double digest RADseq: An inexpensive method for de novo SNP discovery and genotyping in model and non-model species. PLoS One. 2012;7:e37135.

55. Gamble T, Zarkower D. Identification of sex-specific molecular markers using restriction site-associated DNA sequencing. Mol Ecol Resour. 2014;14:902-13.

56. Recknagel $\mathrm{H}$, Elmer KR, Meyer A. A hybrid genetic linkage map of two ecologically and morphologically divergent midas cichlid fishes (Amphilophus spp.) obtained by massively parallel DNA sequencing (ddRADSeq). Genes Genomes Genetics. 2013:3:65-74.

57. Ma XF, Jensen E, Alexandrov N, Troukhan M, Zhang LP, Thomas-Jones S, et al. High resolution genetic mapping by genome sequencing reveals genome duplication and tetraploid genetic structure of the diploid Miscanthus sinensis. PLoS One. 2012;7:e33821.

58. Amores A, Catchen J, Ferrara A, Fontenot Q, Postlethwait JH. Genome evolution and meiotic maps by massively parallel DNA sequencing: Spotted gar, an outgroup for the teleost genome duplication. Genetics. 2011;188:799-808.

59. O'Rourke SM, Yochem J, Connolly AA, Price MH, Carter L, Lowry JB, et al. Rapid mapping and identification of mutations in Caenorhabditis elegans by restriction site-associated DNA mapping and genomic interval pull-down sequencing. Genetics. 2011;189:767-78. 
60. Anderson JL, Mari AR, Braasch I, Amores A, Hohenlohe P, Batzel P, et al. Multiple sex-associated regions and a putative sex chromosome in zebrafish revealed by RAD mapping and population genomics. PLoS One. 2012;7:e40701.

61. Yu H, Xie W, Li J, Zhou F, Zhang Q. A whole-genome SNP array (RICE6K) for genomic breeding in rice. Plant Biotechnol J. 2014;12:28-37.

62. Wang S, Wong D, Forrest $K$, Allen A, Chao S, Huang BE, et al. Characterization of polyploid wheat genomic diversity using a high-density 90000 single nucleotide polymorphism array. Plant Biotechnol J. 2014;12:787-96.

63. Li F, Wang X, Liu J, Fu W, Duan D, Yang Y. Genetic mapping of the Laminaria japonica (Laminarales, Phaeophyta) using amplified fragments lengthpolymorphism markers. J Phycol. 2009;45:1228-33.

64. Yang G, Sun Y, Shi Y, Zhang L, Guo S, Li B, et al. Construction and characterization of a tentative amplified fragment length polymorphism-simple sequence repeat linkage map of Lamianria (Lamianrials, Phaeophyta). J Phycol. 2009;45:873-8.

65. Li Y, Yang Y, Liu J, Wang X, Gao T, Duan D. Genetic mapping of Laminaria japonica and L. longissima using amplified fragment length polymorphism markers in a "two-way pseudo-testcross" strategy. J Integr Plant Biol. 2007:49:392-400.

66. Zhang LN, Peng J, Li XJ, Liu YL, Cui CJ, Wu H, et al. Development of 27 trinucleotide microsatellite markers for Saccharina japonica using next generation sequencing technology. Conserv Genetics Resourc. 2014;6:341-4.

67. Liu F, Wang F, Duan D. EST-SSR markers derived from Laminaria digitata and its transferable application in Saccharina japonica. J Appl Phycol. 2012;24:501-5

68. Liu F, Wang X, Yao J, Fu W, Duan D. Development of expressed sequence tag-derived microsatellite markers for Saccharina (Laminaria) japonica. J Appl Phycol. 2010;22:109-11.

69. Shi Y, Yang G, Liu Y, Liao M, Li X, Cong Y. Development of 18 polymorphic microsatellite DNA markers of Laminaria japonica (Phaeophyceae) Mol Ecol Notes. 2007;7:620-2

70. Liu F, Shao Z, Zhang H, Liu J, Wang X, Duan D. QTL mapping for frond length and width in Laminaria japonica Aresch (Laminarales, Phaeophyta) using AFLP and SR. Marine Biotechnol. 2010;12:386-94.

71. Liu F, Yao J, Wang X, Repnikova A, Galanin DA, Duan D. Genetic diversity and structure within and between wild and cultivated Saccharina japonica (Laminariales, Phaeophyta) revealed by SSR markers. Aquaculture. 2012;358-359:139-45.

72. Li B, Shi Y, Yang G, Che S, Li X, Cong Y. Microsatellite DNA variation of the gametophyte clones isolated from introduced Laminaria japonica (Phaeophyta) and L. lonngissima of China and varieties derived from them J Integr Plant Biol. 2008;50:352-9.

73. Li X, Yang G, Shi Y, Cong Y, Che S, Qu S, et al. Prediction of the heterosis of Laminaria hybrids with the genetic distance between their parental gametophyte clones. J Appl Phycol. 2008;20:1097-102.

74. Cock JM, Sterck L, Rouze P, Scornet D, Allen AE, Amoutzias G, et al. The Ectocarpus genome and the independent evolution of multicellularity in brown algae. Nature. 2010;465:617-21.

75. Catchen JM, Amores A, Hohenlohe P, Cresko W, Postlethwait JH. Stacks: Building and genotyping loci de novo from short-read sequences. Genes Genomes Genetics. 2011;1:171-82.

76. Hohenlohe PA, Bassham S, Etter PD, Stiffler N, Johnson EA, Cresko WA. Population genomics of parallel adaptation in threespine stickleback using sequenced RAD tags. PLoS Genet. 2010;6:e1000862.

77. van Ooijen JW. Multipoint maximum likelihood mapping in a full-sib family of an outbreeding species. Gen Res (Cambridge). 2011;93:343-9.

78. van Ooijen JW, Voorrips RE. JoinMap: software for the calculation of genetic linkage maps, version 3.0. Wageningen, The Netherlands: Plant Research International; 2001

79. Voorrips RE. MapChart: Software for the graphical presentation of linkage maps and QTLs. J Hered. 2002;93:77-8

80. Fishman L, Kelly AJ, Morgan E, Willis JH. A genetic map in the Mimulus guttatus species complex reveals transmission ratio distortion due to heterospecific interactions. Genetics. 2001:159:1701-16.

81. Chakravarti A, Lasher LK, Reefer JE. A maximum likelihood method for estimating genome length using genetic linkage data. Genetics. 1991;128:175-82

82. Zhang L, Yang G, Guo S, Wei Q, Zou G. Construction of a genetic linkage map for silver carp (Hypophthalmichthys molitrix). Anim Genet. 2010;41:523-30

83. Zhou X, Xia Y, Ren X, Chen Y, Huang L, Huang S, et al. Construction of a SNP-based genetic linkage map in cultivated peanut based on large scale marker development using next-generation double digest restriction site associated DNA sequencing (ddRADseq). BMC Genomics. 2014;15:351.

84. Zhang J, Boualem A, Bendahmane A, Ming R. Genomics of sex determination. Curr Opin Plant Biol. 2014;18:110-6.

85. Houston P, Simon PJ, Broach JR. The Saccharomyces cerevisiae recombination enhancer biases recombination during interchromosomal mating-type switching but not in interchromosomal homologous recombination. Genetics. 2004;166:1187-97.

86. Aryal R, Ming R. Sex determination in flowering plants: Papaya as a model system. Plant Sci. 2014;217-218:56-62

87. Wang J, Na J-K, Yu Q, Gschwend AR, Han J, Zeng F, et al. Sequencing papaya $X$ and $Y^{h}$ chromosomes reveals molecular basis of incipient sex chromosome evolution. Proc Natl Acad Sci U S A. 2012:109:13710-5.

88. Gschwend AR, Yu Q, Tong EJ, Zeng F, Han J, VanBuren R, et al. Rapid divergence and expansion of the $\mathrm{X}$ chromosome in papaya. Proc Natl Acad Sci U S A. 2012;109:13716-21

89. de Hoff PL, Ferris P, Olson BJSC, Miyagi A, Geng S, Umen JG. Species and population level molecular profiling reveals cryptic recombination and emergent asymmetry in the dimorphic mating locus of $C$. reinhardtii. PLoS Genet. 2013;9:e1003724.

90. Goodenough U, Lin H, Lee J-H. Sex determination in Chlamydomonas. Semin Cell Dev Biol. 2007:18:350-61.

91. Umen JG. Evolution of sex and mating loci: an expanded view from Volvocine algae. Curr Opin Microbiol. 2011;14:634-41.

92. Ferris P, Olson BJSC, de Hoff PL, Douglass S, Casero D, Prochnik S, et al Evolution of an expanded sex-determining locus in Volvox. Science. 2010;238:351-4.

93. Telgmann-Rauber A, Jamsari A, Kinney MS, Pires JC, Jung C. Genetic and physical maps around the sex-determining M-locus of the dioecious plant asparagus. Molecular Genetics \& Genomics. 2007;278:221-34.

94. Yamato KT, Ishizaki K, Fujisawa M, Okada S, Nakayama S, Fujishita M, et al. Gene organization of the liverwort $Y$ chromosome reveals distinct sex chromosome evolution in a haploid system. Proc Natl Acad Sci U S A. 2007; 104:6472-7

95. McDaniel SF, Willis JH, Shaw AJ. A linkage map reveals a complex basis for segregation distortion in an interpopulation cross in the moss Ceratodon purpureus. Genetics. 2007;176:2489-500.

96. Frenkel J, Vyverman W, Pohnert G. Pheromone signaling during sexual reproduction in algae. Plant J. 2014:79:632-44.

97. Lane CE, Archibald JM. The eukaryotic tree of life: Endosymbiosis takes its TOL. Trends Ecol Evol. 2008:23:268-75.

98. Koonin EV. The incredible expanding ancestor of eukaryotes. Cell. 2010;140:606-8.

99. Ahmed S, Cock JM, Pessia E, Luthringer R, Cormier A, Robuchon M, et al. A haploid system of sex determination in the brown alga Ectocarpus sp. Curr Biol. 2014;24:1945-57.

\section{Submit your next manuscript to BioMed Central and take full advantage of:}

- Convenient online submission

- Thorough peer review

- No space constraints or color figure charges

- Immediate publication on acceptance

- Inclusion in PubMed, CAS, Scopus and Google Scholar

- Research which is freely available for redistribution 